\title{
Identification for Active Vibration Control of Flexible Structure Based on Prony Algorithm
}

\author{
Xianjun Sheng, ${ }^{1}$ Yuanli Kong, ${ }^{1}$ Fengyun Zhang, ${ }^{2}$ and Rui Yang ${ }^{3}$ \\ ${ }^{1}$ School of Electrical Engineering, Dalian University of Technology, Dalian 116024, China \\ ${ }^{2}$ North Automatic Control Technology Institute, Taiyuan 030006, China \\ ${ }^{3}$ School of Mechanical Engineering, Dalian University of Technology, Dalian 116024, China \\ Correspondence should be addressed to Yuanli Kong; kongyuanli@mail.dlut.edu.cn
}

Received 5 June 2016; Accepted 3 August 2016

Academic Editor: Mickaël Lallart

Copyright (C) 2016 Xianjun Sheng et al. This is an open access article distributed under the Creative Commons Attribution License, which permits unrestricted use, distribution, and reproduction in any medium, provided the original work is properly cited.

\begin{abstract}
Flexible structures have been widely used in many fields due to the advantages of light quality, small damping, and strong flexibility. However, flexible structures exhibit the vibration in the process of manipulation, which reduces the pointing precision of the system and causes fatigue of the machine. So, this paper focuses on the identification method for active vibration control of flexible structure. The modal parameters and transfer function of the system are identified from the step response signal based on Prony algorithm, while the vibration is attenuated by using the input shaping technique designed according to the parameters identified from the Prony algorithm. Eventually, the proposed approach is applied to the most common flexible structure, a piezoelectric cantilever beam actuated by Macro Fiber Composite (MFC). The experimental results demonstrate that the Prony algorithm is very effective and accurate on the dynamic modeling of flexible structure and input shaper could significantly reduce the vibration and improve the response speed of system.
\end{abstract}

\section{Introduction}

Flexible structures experience undesirable vibration in response to environmental and operational forces. The very existence of vibrations not only can limit the accuracy of sensitive instruments or cause significant error in applications, where high positioning precision is essential, but also plays an important role in causing component fatigue and shortening the life of the system [1-3]. Therefore, vibration control of flexible structures has attracted a considerable amount of investigations in many scientific and engineering applications.

The vibration of flexible structure is a quite complicated issue concerning many factors and the key technique is to obtain an accurate dynamic model of the system. Therefore, a variety of studies have been done on dynamic modeling for vibration control of flexible structures. An analytic modeling method based on Hamilton's principles is presented in [4] to procure the dynamic equation of flexible beam and the independent modal method by using piezo-patches as actuators is utilized to realize the vibration suppression. However, when using the analytic modeling, the detailed physical information regarding the whole system is required and the descriptive partial differential equations must be solved, which makes it very difficult to obtain model parameters. It seems that finite element is a better approach compared with analytic model because there is no requirement to solve a partial differential equation [5-7]. Unfortunately, detailed information regarding the materials of structure and boundary conditions is still required. Many efforts were directed towards system identification which is a straightforward method to obtain a structural model from experimental data. An identification method on procuring transfer function from time domain data can be found in $[8,9]$ and literature [10] put forward a frequency identification approach by swept frequency analysis. And, recently, artificial intelligence techniques are used in system identification for flexible structure, such as genetic algorithm [11] and neural networks $[12,13]$. The previous identification methods exhibit good performances on dynamic modeling for flexible structure, but they failed to identify both modal parameters and transfer 
TABLE 1: The main parameters of piezoelectric cantilever beam.

\begin{tabular}{lcccccc}
\hline Material & Young's modulus $(\mathrm{GPa})$ & Density $\left(\mathrm{Kg} / \mathrm{m}^{3}\right)$ & Poisson's ratio & Length $(\mathrm{mm})$ & Width $(\mathrm{mm})$ & Thickness $(\mathrm{mm})$ \\
\hline Aluminum layer & 69 & 2800 & 0.34 & 150 & 13 & 1 \\
Bonding layer & 2.5 & 2700 & 0.36 & 101 & 13 & 0.1 \\
MFC & 30 & 7450 & 0.31 & 101 & 13 & 0.3 \\
\hline
\end{tabular}

function simultaneously. Therefore, in this paper, a new identification method based on Prony algorithm is proposed to establish the system dynamic model and combines the input shaping technique to achieve an active vibration control for flexible structure. Prony algorithm uses a linear combination of complex exponential functions to describe equally spaced sampling data and calculates the frequency, amplitude, damping factor, and initial phase of the oscillation system from the response signal. Prony algorithm has succeeded in estimating the modal parameters of power system oscillations and hydro turbine generating sets, referencing articles [1417], but Prony algorithm has never been used in vibration analysis for flexible structures. So, this paper identifies the modal parameters and transfer function for flexible structure based on Prony algorithm. Then the identification results combine the input shaping techniques to achieve an active vibration control for a piezoelectric cantilever beam.

The organization of this paper is as follows. Section 2 presents a piezoelectric cantilever beam which is considered as flexible structure in detail and analyzes the system's characteristic. Section 3 introduces Prony algorithm on identifying the modal parameters and transfer function for flexible structure. Section 4 gives details on control method and the design of input shaper based on identification results. Section 5 presents experimental results on the proposed identification and vibration control. Section 6 summarizes the paper.

\section{Description and Analysis of the Flexible Structure Behavior}

A piezoelectric cantilever beam actuated by Macro Fiber Composites (MFC) is used as flexible structure in this paper. The beam is cantilevered at one end and a MFC patch used as an actuator is bonded on the surface of aluminum layer (see Figure 1). The MFC composed of resin and a piezoelectric ceramic with $0^{\circ}$ fiber orientation which can offer higher performance, durability, and flexibility compared with piezoelectric ceramics. The MFC used in this paper is developed by NASA and its model is M-8507-P1. The workspace of MFC is $85 \mathrm{~mm} \times 7 \mathrm{~mm}$ and free strain epsilon is $1380 \mathrm{pzpm}$. The main characteristics of the piezoelectric cantilever beam are presented in Table 1.

The whole experimental framework is shown in Figure 2. The computer with software LabVIEW is used as the controller and the data acquisition (DAQ) card whose model is NI USB- 6229 is used for digital-to-analogue (D/A) and analogue-to-digital (A/D) conversions. A voltage amplifier with a fixed gain 200 is used to provide driving voltage for the

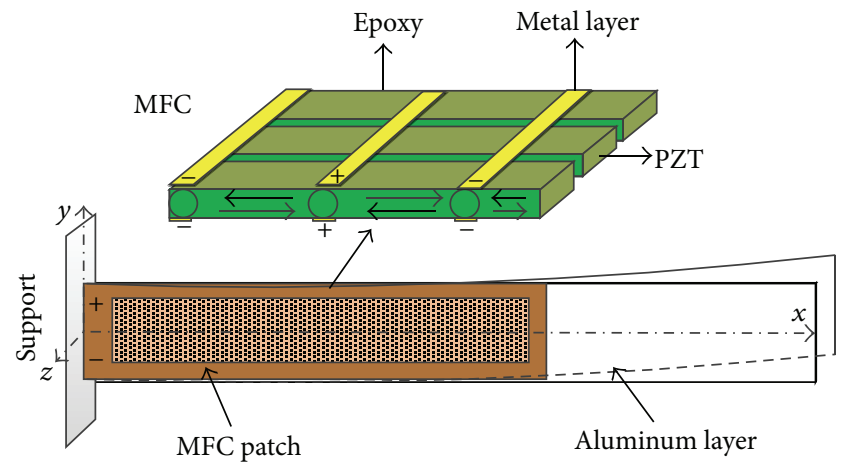

FIgURE 1: The structure of a piezoelectric cantilever actuated by MFC.

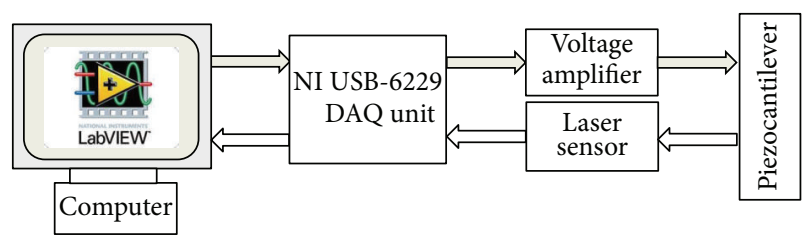

FIGURE 2: The schematic of experiment platform.

MFC. In order to detect the displacement of the piezocantilever and validate the experimental performances, a laser sensor from KEYENCE with an accuracy of $1 \mu \mathrm{m}$ and a high passband is utilized.

When a step input voltage is applied, the oscillation emerges prominently at the free end of piezoelectric cantilever and attenuates gradually with the time. The existence of oscillation severely degrades the positioning accuracy and increases the settling time. In this paper, the dynamic modeling of piezoelectric cantilever is assumed as linear time-invariant, without consideration of hysteresis and creep. In this paper, the behavior of piezoelectric cantilever is modeled based on the Prony algorithm and the vibration is suppressed by input shaping technique. The whole schematic of identification for active vibration control is depicted in Figure 3. The modeling and vibration control are performed step by step. These steps are as follows. Firstly, the transfer function and modal parameters of the system are identified through Prony algorithm by applying the excitation signal to the controlled object. Then the input shaper is designed based on the previously obtained parameters. At last, the reference input signal is convoluted with the impulse sequences gained from the input shaper to realize the vibration suppression. 


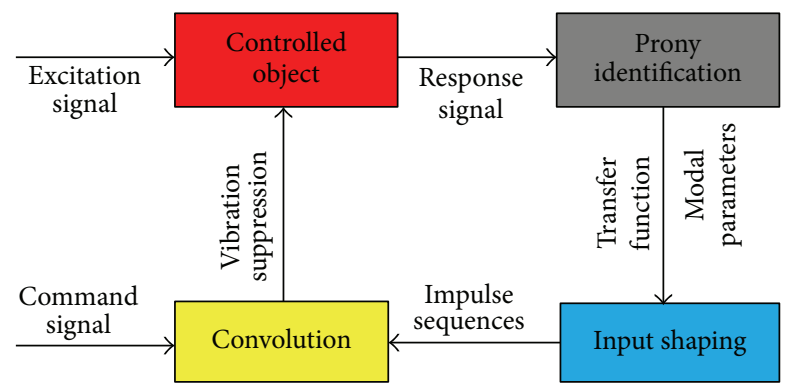

FIgURE 3: The schematic of identification for active vibration control.

\section{Modal Parameters and Transfer Function Identification Based on Prony Algorithm}

In this section, an identification method based on Prony algorithm is proposed for a flexible structure with $n$ modes. After using the proposed scheme to the flexible system, the modal parameters and transfer function of the system can be obtained effectively.

3.1. The Prony Algorithm. Prony analysis supposes that the model is a linear combination of a series of exponential functions which have arbitrary amplitude, phase, frequency, and damping factor and then uses this model to fit equally spaced sampling data to determine corresponding parameters. The general expression of the discrete time signal is as follows:

$$
\widehat{x}(n)=\sum_{i=1}^{p} b_{i} z_{i}^{n}, \quad n=0,1, \ldots, N-1,
$$

where $b_{i}=A_{i} \exp \left(j \theta_{i}\right), z_{i}=\exp \left[\left(a_{i}+j 2 \pi f_{i}\right) \Delta t\right], p$ is the number of exponential functions, $A_{i}$ is the $i$ th amplitude of the exponential function, $\theta_{i}$ is the primary phase, $f_{i}$ is the frequency, $\alpha_{i}$ is the damping coefficient, and $\Delta t$ is the sampling interval. The process and steps of solving $b_{i}$ and $z_{i}$ and the values of the amplitude, phase, frequency, and damping factor by Prony algorithm were detailed in literature $[18,19]$. In order to extract more characteristics of the system, this paper employs the multiple Prony models that differ from the single Prony model in the way of determining the sample function matrix, amplitude, and phase of each model. The main steps of the Prony algorithm are given as follows.

Step 1. Calculate the sample function $r(i, j)$ and construct the matrix $\mathbf{R}_{\mathbf{e}}$ by the following:

$$
\mathbf{R}_{\mathbf{e}}=\left[\begin{array}{cccc}
r(1,0) & r(1,1) & \cdots & r(1, p) \\
r(2,0) & r(2,1) & \cdots & r(2, p) \\
\vdots & \vdots & \vdots & \vdots \\
r\left(p_{e}, 0\right) & r\left(p_{e}, 1\right) & \cdots & r\left(p_{e}, p_{e}\right)
\end{array}\right] \quad\left(p_{e} \geq p\right)
$$

where $r(i, j)=\sum_{n=p_{e}}^{N-1} x(n-j) x^{*}(n-i), i, j=0,1, \ldots, p_{e}$.
Step 2. Use the Singular Value Decomposition and Total Least Squares (SVD-TLS) algorithm to determine the rank of $\mathbf{R}_{\mathrm{e}}$.

Step 3. Solve the linear equation (3) to obtain $a_{1}, \ldots, a_{p}$ by the least squares estimation:

$$
\left[\begin{array}{cccc}
r(0,0) & r(0,1) & \cdots & r(0, p) \\
r(1,0) & r(1,1) & \cdots & r(1, p) \\
\vdots & \vdots & \cdots & \vdots \\
r(p, 0) & r(p, 1) & \cdots & r(p, p)
\end{array}\right]\left[\begin{array}{c}
1 \\
a_{1} \\
\vdots \\
a_{p}
\end{array}\right]=\left[\begin{array}{c}
\varepsilon(p) \\
0 \\
\vdots \\
0
\end{array}\right] .
$$

Step 4. Calculate the roots of characteristic polynomial $1+$ $a_{1} z^{-1}+\cdots+a_{p} z^{-p}=0$ and compute $\hat{x}(n)$ according to recursive formula $\widehat{x}(n)=-\sum_{i=1}^{p} a_{i} \widehat{x}(n-i)$.

Step 5. Calculate parameter $b_{i}$ according to the following:

$$
\left[\begin{array}{cccc}
1 & 1 & \cdots & 1 \\
z_{1} & z_{2} & \cdots & z_{p} \\
\vdots & \vdots & \vdots & \vdots \\
z_{1}^{N-1} & z_{2}^{N-1} & \cdots & z_{p}^{N-1}
\end{array}\right]\left[\begin{array}{c}
b_{1} \\
b_{2} \\
\vdots \\
b_{p}
\end{array}\right]=\left[\begin{array}{c}
\widehat{x}(0) \\
\widehat{x}(1) \\
\vdots \\
\hat{x}(N-1)
\end{array}\right] .
$$

Step 6. Calculate amplitude $A_{i}$, phase $\theta_{i}$, frequency $f_{i}$, and damping coefficient $\alpha_{i}$ according to the following:

$$
\begin{aligned}
A_{i} & =\left|b_{i}\right|, \\
\theta_{i} & =\frac{\arctan \left[\operatorname{Im}\left(b_{i}\right) / \operatorname{Re}\left(b_{i}\right)\right]}{(2 \pi \Delta t)}, \\
\alpha_{i} & =\frac{\ln \left|z_{i}\right|}{\Delta t}, \\
f_{i} & =\frac{\arctan \left[\operatorname{Im}\left(z_{i}\right) / \operatorname{Re}\left(z_{i}\right)\right]}{(2 \pi \Delta t)} .
\end{aligned}
$$

3.2. Transfer Function Identifying Based on Prony Algorithm. In this section, considering a Single Input Single Output (SISO) linear system, $I(s)$ and $Y(s)$, respectively, stand for input signal and output signal in Laplace transform and $Y(s)=I(s) G(s)$; then we suppose that the system transfer function $G(s)$ can be expressed as

$$
G(s)=\sum_{i=1}^{n} \frac{R_{i}}{s-\lambda_{i}},
$$

where $n$ is the order of the model and $R_{i}$ is the residue of transfer function in pole $\lambda_{i}$. Assuming that the input signal consists of a series of delayed signals, then $I(s)$ can be expressed as

$$
I(s)=\frac{c_{0}+c_{1} e^{-s D_{1}}+c_{2} e^{-s D_{2}}+\cdots+c_{k} e^{-s D_{k}}}{s-\lambda_{n+1}},
$$

where $c_{i}(i=0,1, \ldots, k)$ is delay factor and $D_{j}(j=1, \ldots, k)$ is characteristic value. 
When an input signal $I(s)$ is applied to the system, the output signal $Y(s)$ can be expressed as

$$
\begin{aligned}
& Y(s) \\
& \quad=\left(c_{0}+c_{1} e^{-s D_{1}}+\cdots+c_{k} e^{-s D_{k}}\right) \sum_{i=1}^{n} \frac{R_{i}}{\left(s-\lambda_{i}\right)\left(s-\lambda_{n+1}\right)} .
\end{aligned}
$$

By partial fraction method, (8) can be transformed into

$$
\begin{array}{r}
Y(s)=\left(c_{0}+c_{1} e^{-s D_{1}}+\cdots+c_{k} e^{-s D_{k}}\right) \\
\cdot\left[\frac{Q_{n+1}}{\left(s-\lambda_{n+1}\right)}+\sum_{i=1}^{n} \frac{Q_{i}}{\left(s-\lambda_{i}\right)}\right],
\end{array}
$$

where $Q_{i}=R_{i} /\left(\lambda_{i}-\lambda_{n+1}\right), i=1,2, \ldots, n$, and $Q_{n+1}=$ $-\sum_{i=1}^{n} Q_{i}$. Then the Laplace inverse transform of $Y(s)$ can be shown as

$$
\begin{aligned}
Y(t)= & c_{0}\left(Q_{n+1} e^{\lambda_{n+1} t}+\sum_{i=1}^{n} Q_{i} e^{\lambda_{i} t}\right) u(t) \\
& +\sum_{i=1}^{k} c_{i}\left(Q_{n+1} e^{\lambda_{n+1}\left(t-D_{i}\right)}+\sum_{i=1}^{n} Q_{i} e^{\lambda_{i}\left(t-D_{i}\right)}\right) \\
& \cdot u\left(t-D_{i}\right) .
\end{aligned}
$$

In order to apply the Prony method, let $\tau=t-D_{k}$ and $v(\tau)=$ $y\left(t+D_{k}\right)$; then (10) can be expressed in the form

$$
v(\tau)=\sum_{j=1}^{n+1} B_{j} e^{\lambda_{i} \tau}
$$

where

$$
B_{j}=Q_{j}\left(\sum_{i=0}^{k} c_{i} e^{\lambda_{j}\left(D_{k}-D_{i}\right)}\right) \quad(j=1,2, \ldots, n+1) .
$$

When $n, \lambda_{i}$, and $R_{i}$ in (6) are available, the system transfer function can be obtained. Residues are shown, respectively, as

$$
R_{j}=\frac{B_{j}\left(\lambda_{j}-\lambda_{n+1}\right)}{\sum_{i=0}^{k} c_{i} e^{\lambda_{j}\left(D_{k}-D_{i}\right)}}, \quad j=1,2,3, \ldots, n .
$$

\section{Input Shaper Design for Vibration Control}

A number of techniques have been proposed to suppress the vibration, such as feedback control [20], positive position feedback (PPF) [21], and input shaper [22]. Both closed-loop feedback technique and PPF use the system's state to reduce the vibration. However, the input shaping is implemented by convolving a sequence of impulses with a desired system command to produce a shaped input to drive the actuator to reduce the system oscillations [23]. Furthermore, only the modal parameters are required for the design of input shaper and these parameters can be obtained from Prony algorithms presented in Section 3. So, the input shaping technique is utilized to attenuate the oscillations in this paper.
Input shaper is a time-delay filter that convolves the command input signal with a series of impulse sequences based on the natural frequencies and damping coefficients of the system. If the timing and amplitudes of impulses are chosen to match modal parameters of the controlled system, vibration can be eliminated [24]. The basic structure of input shaper can be expressed as

$$
C(s)=\sum_{i}^{n} A_{i} e^{-s t_{i}}
$$

where $A_{i}$ is the amplitude of the impulse, $t_{i}$ is the time of the impulse input, and $n$ is the number of impulse sequences.

The derivation of the input shaper is based on the linear system theory. An arbitrary linear vibrating system can be specified as a cascaded multiple second-order oscillation system, so the analysis of the original system can be realized by studying a second-order system. A second-order oscillation system with natural frequency $\omega_{n}$ and damping coefficient $\xi(0<\xi<1)$ can be expressed as

$$
G(s)=\frac{\omega_{n}^{2}}{s^{2}+2 \xi \omega_{n} s+\omega_{n}^{2}} .
$$

The unit impulse response of a second-order system can be written as

$$
y(t)=\frac{\omega_{n}}{\sqrt{1-\xi^{2}}} e^{-\xi \omega_{n}} \sin \omega_{d}\left(t-t_{i}\right)
$$

where $\omega_{d}=\omega_{n} \sqrt{1-\xi^{2}}$. When input shaper is applied to a controlled system, the impulse response of this system can be written as

$$
y(t)=\sum_{i=1}^{n} \frac{\omega_{n} A_{i}}{\sqrt{1-\xi^{2}}} e^{-\xi \omega_{n}} \sin \omega_{d}\left(t-t_{i}\right) .
$$

Define the ratio of the amplitude of (17) and (16) as vibration of the system:

$$
V\left(\omega_{n}, \xi\right)=e^{-\xi \omega_{n} t_{n}} \sqrt{C^{2}\left(\omega_{n}, \xi\right)+S^{2}\left(\omega_{n}, \xi\right)},
$$

where

$$
\begin{aligned}
& C\left(\omega_{n}, \xi\right)=\sum_{i=1}^{n} A_{i} e^{-\xi \omega_{n} t_{n}} \cos \left(\omega_{n} \sqrt{1-\xi^{2}} t_{i}\right) \\
& S\left(\omega_{n}, \xi\right)=\sum_{i=1}^{n} A_{i} e^{-\xi \omega_{n} t_{n}} \sin \left(\omega_{n} \sqrt{1-\xi^{2}} t_{i}\right) .
\end{aligned}
$$

In order to make the vibration equal to zero, $C\left(\omega_{n}, \xi\right)=0$ and $S\left(\omega_{n}, \xi\right)=0$ are prerequisite. When adding different constraints, diverse input shapers can be designed. The zero vibration input shaper (ZV shaper) is the simplest shaper, consisting of two impulses. In order to shorten the time of shaping, we can set $t_{1}=0$ and let $A_{i}>0$ and $\sum_{i=1}^{n} A_{i}=1$ to achieve the set point of system. Hence, the constraints of 


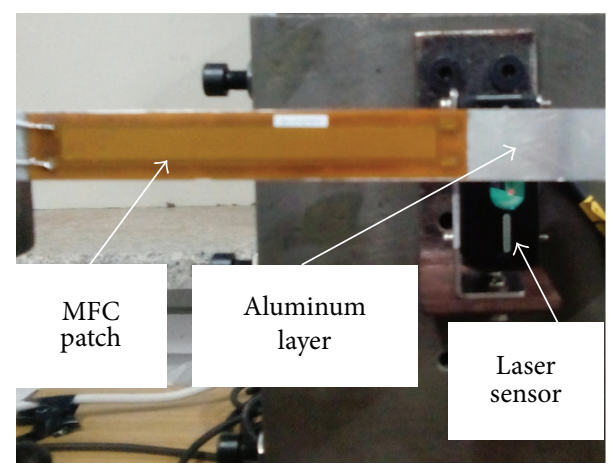

FIGURE 4: The experiment setup.

$\mathrm{ZV}$ input shaper are expressed as $A_{1}+A_{2}=1, A_{1}>0$, and $A_{2}>0$; the solutions can be written as

$$
\begin{array}{ll}
A_{1}=\frac{1}{1+K}, & t_{1}=0, \\
A_{2}=\frac{K}{1+K}, & t_{2}=T,
\end{array}
$$

where $K=e^{-\xi \pi / \sqrt{1-\xi^{2}}}$ and $T=\pi / \omega_{n} \sqrt{1-\xi^{2}}$. When the identified modal parameters cannot fit the actual system parameters well, ZV shaper cannot eliminate the vibration effectively. In order to improve the robustness of the system, a constraint $(d / d \omega) V(\omega, \xi)=0$ is applied to configure multiple poles for the system and then a zero vibration and derivative (ZVD) shaper with three impulses can be solved. The parameters of ZVD shaper can be expressed as

$$
\begin{array}{ll}
A_{1}=\frac{1}{1+2 K+K^{2}}, & t_{1}=0, \\
A_{2}=\frac{2 K}{1+2 K+K^{2}}, & t_{2}=T, \\
A_{3}=\frac{K^{2}}{1+2 K+K^{2}}, & t_{3}=2 T .
\end{array}
$$

\section{Experiment Results}

In order to verify the feasibility of the identification algorithm and test the effect of the vibration control strategy, a piezoelectric cantilever beam described in Section 2 is used to establish the experimental platform. The excitation signal is programmed by software LabVIEW. This generated signal is output through the D/A conversion module of data acquisition card whose analog output range is $-10 \mathrm{~V} \sim+10 \mathrm{~V}$ and then the signal is amplified by a voltage amplifier to actuate the MFC. Finally, the displacement of piezoelectric cantilever beam is monitored by a laser sensor put at the tip vertex point of the beam at the free end and recorded at $1 \mathrm{kHz}$ via an $\mathrm{A} / \mathrm{D}$ converter. The experiment setup is shown in Figure 4.

5.1. The Identification Results Based on Prony Algorithm. A $1 \mathrm{~V}$ step signal as excitation signal is applied to piezoelectric cantilever beam system and output displacement of the beam is acquired as identification signal data with the sampling interval of $5 \mathrm{~ms}$ in this section. The $1 \mathrm{~V}$ step signal is expressed as $c_{0}=1, \lambda_{n+1}=0$, and $c_{i}=0(i=1, \ldots, k)$ according to (7). When the step signal lasts for a long time, the creep will engender, so the whole sampling time is chosen as $1 \mathrm{~s}$ to avoid the influence of creep effect on the identification. In general, it is inevitable that the noise exists in the measurement of displacement via a sensor, but, unfortunately, the Prony algorithm is sensitive to the data nosing. Hence, the wavelet threshold denoising is used to preprocess the measuring response signal and improve signal-to-noise ratio before using Prony algorithm. The result of filtering is shown in Figure 5. Furthermore, direct current component could lead to poor identification precision when using the Prony algorithm, so it is imperative to extract direct current component from the signal data. The direct current component is $0.195 \mathrm{~mm}$ via calculation. Then the response signal with filtering and removing the direct current component is used as identification data and the identification results based on Prony algorithm are shown in Figure 6(a). The identification error is shown in Figure 6(b) and the root mean square error (RMSE) of identification is $0.005 \mathrm{~mm}$. The main parameters obtained from identification results are shown in Table 2.

From Figure 6, the fitting signal by Prony algorithm can describe the actual experimental data exactly, which could illustrate that Prony algorithm has active effects on dynamic modeling for flexible structure. As shown in Table 2, the order of the system is 5, including a first-order inertial element and two second-order oscillation subsystems. Oscillation mode contains coneigenvalues 1 and $2(44.209 \mathrm{~Hz})$ and coneigenvalues 4 and $5(19.112 \mathrm{~Hz})$. It is obvious that the oscillation mode 1 and mode 2 attenuate rapidly because the damping factor of them is relatively large and oscillation amplitude is relatively small; therefore the dominant oscillation frequency is $19.112 \mathrm{~Hz}$. The system transfer function can be expressed as (22) and frequency response curve is shown in Figure 7.

$$
G(s)=\frac{1.022 s^{4}+3474 s^{3}+3.233 \times 10^{5} s^{2}+2.004 \times 10^{8} s+1.063 \times 10^{9}}{s^{5}+99.33 s^{4}+9.439 \times 10^{4} s^{3}+1.987 \times 10^{6} s^{2}+1.15 \times 10^{9} s+5.185 \times 10^{9}}
$$

5.2. Experimental Validation of Performance of Input Shaper. The transfer function and modal parameters obtained from the Prony algorithm are used directly to design the input shaper in this section. The dominant modal parameters are 
TABLE 2: Identification results on system parameters.

\begin{tabular}{lccccc}
\hline Order & $A_{i}$ & $f_{i} / \mathrm{Hz}$ & $\alpha_{i}$ & $\lambda_{i}$ & $R_{i}$ \\
\hline 1 & 0.00672 & 44.209 & -45.898 & $-45.898+277.754 i$ & $-0.066-1.891 i$ \\
2 & 0.00672 & 44.209 & -45.898 & $-45.898-277.754 i$ & $-0.066-1.891 i$ \\
3 & 0.03093 & 0 & -4.536 & -4.536 & 0.140 \\
4 & 0.08045 & 19.112 & -1.4967 & $-1.497+120.087 i$ & $0.506-9.649 i$ \\
5 & 0.08045 & 19.112 & -1.4967 & $-1.497-120.087 i$ & $0.506+9.649 i$ \\
\hline
\end{tabular}

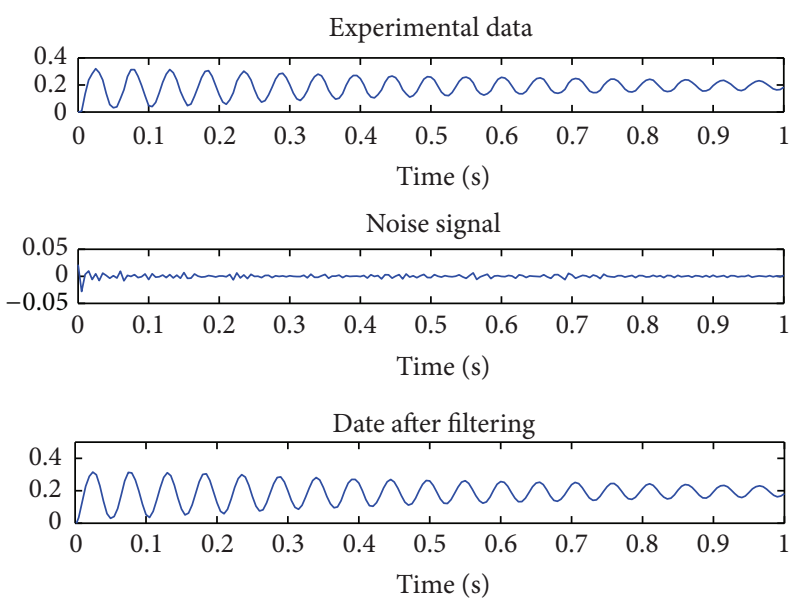

FIGURE 5: The result of signal filtering preprocessing.

shown as natural frequency $\omega_{n}=120.10 \mathrm{rad} / \mathrm{s}$ and damping ratio $\xi=0.01246$. According to (20), the parameters of $\mathrm{ZV}$ input shaper for dominant modal are as follows:

$$
\begin{aligned}
& A_{1}=0.5098, \quad t_{1}=0, \\
& A_{2}=0.4902, \quad t_{2}=0.02616 .
\end{aligned}
$$

And, according to (21), the parameters of ZVD input shaper for dominant modal are as follows:

$$
\begin{array}{ll}
A_{1}=0.2599, & t_{1}=0, \\
A_{2}=0.4998, & t_{2}=0.02616, \\
A_{3}=0.2403, & t_{3}=0.05232 .
\end{array}
$$

Figure 8 shows the experimental results of the vibration control effect under a $1 \mathrm{~V}$ step signal by using $\mathrm{ZV}$ shaper and ZVD shaper compared with the one without shaper. It can be concluded that the input shaper has good effect on suppressing vibration and the ZVD shaper is superior to ZV shaper. Under the effect of ZVD input shaper, the settling time is shortened distinctly from $2 \mathrm{~s}$ to $0.5 \mathrm{~s}$ and the overshoot is null. Furthermore, it can also adequately illustrate that the identification results based on Prony algorithm are effective and accurate.

\section{Conclusion}

This paper proposes an identification method combining with input shaping technique to suppress the vibrations of the flexible system for precise positioning. The dynamic modeling and vibration control are performed in sequence. The dynamic model of the system is considered as a linear time-invariant system, and the Prony algorithm is used to identify the modal parameters and transfer function of the system from the step response signal. In order to improve the accuracy of identification, the data nosing is filtered and the direct current component is removed. The identification results show that the Prony algorithm is quite accurate and effective. The RMSE of identification error is only $0.005 \mathrm{~mm}$ and the identified transfer function with five modes can describe the system's characteristic exactly. The first-order vibration mode is analyzed as $19.112 \mathrm{~Hz}$ from the identification results and then the input shaper is designed to succeed an active vibration control. The experimental results show that the vibration is reduced distinctly and the settling time is also shortened significantly after applying the input shaper.

The works presented in this paper have confirmed that the Prony algorithm combining with input shaping technique can exhibit admirable performance on identification for active vibration control. Such approach is of great interest because it is easy to implement and the vibration is reduced in open loop. What is more, the identification method presented in this paper is well adopted not only for flexible structure but also for other similar manipulation mechanisms and the identified transfer function can also be used to simulate the system and design other vibration controllers. Finally, the future work will focus on the robustness of the controller considering the parameter variations and environmental disturbance. 


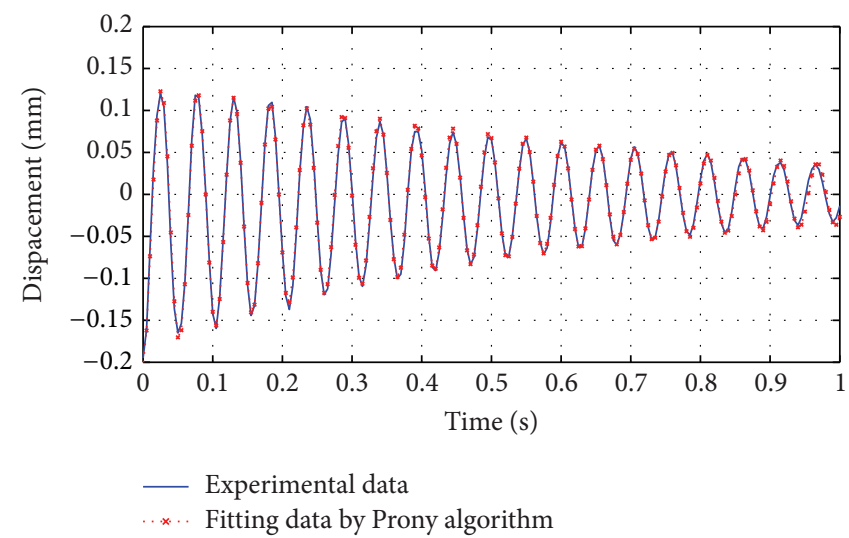

(a) The fitting result by Prony

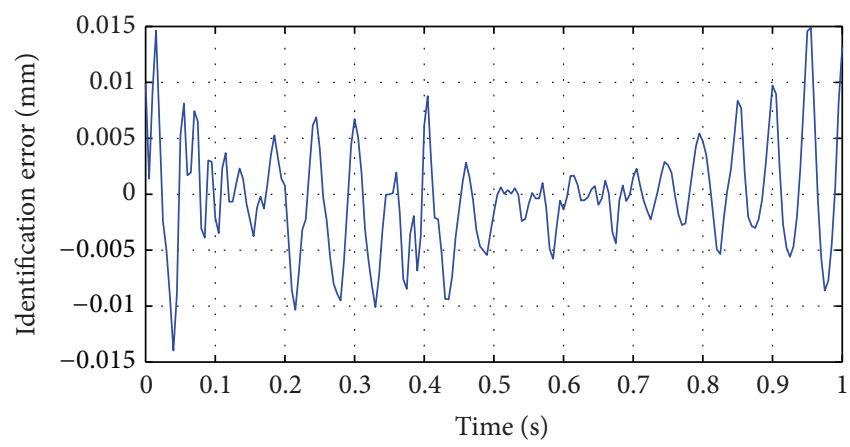

(b) The error of identification

Figure 6: The results of Prony identification.
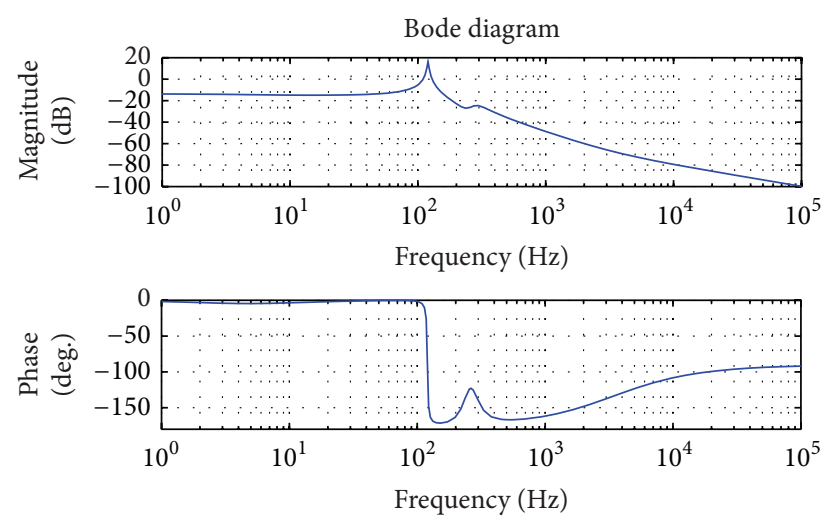

FIgURE 7: The bode diagram of identified system.

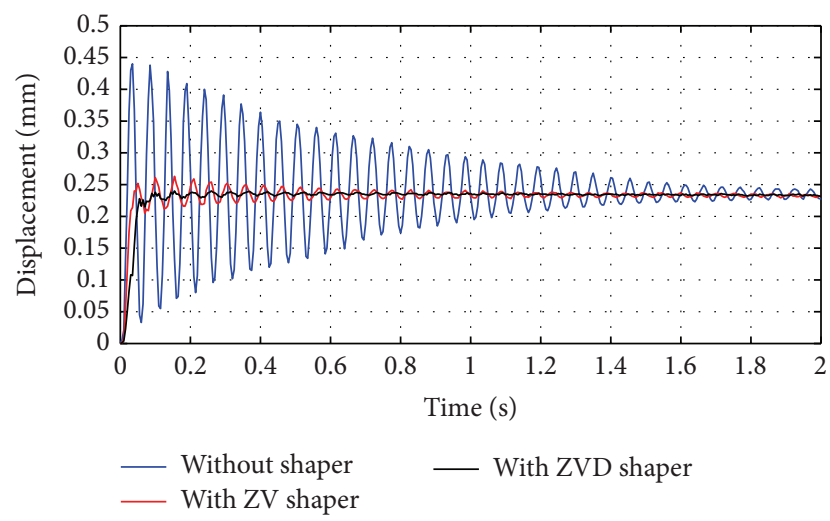

FIGURE 8: Step response of the system with vibration suppression.

\section{Competing Interests}

The authors declare that they have no competing interests.

\section{Acknowledgments}

The authors greatly appreciate the financial support of the National Natural Science Foundation of China (nos. 51275073 and 51321004).

\section{References}

[1] S. O. R. Moheimani and A. J. Fleming, Piezoelectric Transducers for Vibration Control and Damping, Advances in Industrial Control, Springer, 2010.

[2] A. P. Parameswaran, A. B. Pai, P. K. Tripathi, and K. V. Gangadharan, "Active vibration control of a smart cantilever beam on general purpose operating system," Defence Science Journal, vol. 63, no. 4, pp. 413-417, 2013.

[3] Z. Long and D. Guangren, "A robust vibration suppression controller design for space flexible structures," in Proceedings of the 24th Chinese Control and Decision Conference (CCDC '12), vol. 2, pp. 4020-4024, IEEE, Taiyuan, China, 2012.

[4] D. Wu, L. Huang, B. Pan, Y. Wang, and S. Wu, "Experimental study and numerical simulation of active vibration control of a highly flexible beam using piezoelectric intelligent material," Aerospace Science \& Technology, vol. 37, pp. 10-19, 2014.

[5] A. R. Tavakolpour, M. Mailah, I. Z. Mat Darus, and O. Tokhi, "Self-learning active vibration control of a flexible plate structure with piezoelectric actuator," Simulation Modelling Practice and Theory, vol. 18, no. 5, pp. 516-532, 2010.

[6] A. Yousefi-Koma, D. G. Zimcik, and A. Mander, "Experimental and theoretical system identification of flexible structures with piezoelectric actuators," in Proceedings of the 24th Congress of the International Council of the Aeronautical Sciences, Yokohama, Japan, 2004.

[7] Z.-C. Qiu, H.-X. Wu, and C.-D. Ye, "Acceleration sensors based modal identification and active vibration control of flexible smart cantilever plate," Aerospace Science and Technology, vol. 13, no. 6, pp. 277-290, 2009.

[8] M. Viberg, "Subspace-based methods for the identification of linear time-invariant systems," Automatica, vol. 31, no. 12, pp. 1835-1851, 1995.

[9] P. Van Overschee and B. De Moor, "Continuous-time frequency domain subspace system identification," Signal Processing, vol. 52, no. 2, pp. 179-194, 1996.

[10] Y. Wang, S. Zhu, M. Li, K. Miao, and W. Xu, "Frequency domain identification for active vibration control of flexible structures," in Proceedings of the International Conference on Control and Automation, vol. 2, pp. 1149-1153, Budapest, Hungary, June 2005.

[11] A. R. Tavakolpour, I. Z. Mat Darus, O. Tokhi, and M. Mailah, "Genetic algorithm-based identification of transfer function 
parameters for a rectangular flexible plate system," Engineering Applications of Artificial Intelligence, vol. 23, no. 8, pp. 1388-1397, 2010.

[12] R. Ismail, A. Y. Ismail, and I. Z. M. Darus, "Identification algorithms of flexible structure using neural networks," in Proceedings of the 4th Student Conference on Research and Development, pp. 162-168, Selangor, Malaysia, June 2006.

[13] I. Z. M. Darus and M. O. Tokhi, "Soft computing-based active vibration control of a flexible structure," Engineering Applications of Artificial Intelligence, vol. 18, no. 1, pp. 93-114, 2005.

[14] J. Yan, Y. W. Lan, W. Fang, X. Hou, and J. C. Mei, "Prony algorithm's application on extracting the parameters of the power system transient fault," Applied Mechanics and Materials, vol. 602-605, pp. 3877-3880, 2014.

[15] D. P. Wadduwage, U. D. Annakkage, and K. Narendra, "Identification of dominant low-frequency modes in ring-down oscillations using multiple Prony models," IET Generation, Transmission and Distribution, vol. 9, no. 15, pp. 2206-2214, 2015.

[16] Y. J. Tang, Y. J. Tang, and X. L. Xie, “The new identification method for low frequency oscillation mode in power system based on prony algorithm and neural network," Applied Mechanics and Materials, vol. 321-324, pp. 1400-1404, 2013.

[17] D. A. Pierre, D. J. Trudnowski, and J. F. Hauer, "Identifying linear reduced-order models for systems with arbitrary initial conditions using Prony signal analysis," IEEE Transactions on Automatic Control, vol. 37, no. 6, pp. 831-835, 1992.

[18] R. Kumaresan, D. W. Tufts, and L. L. Scharf, "A prony method for noisy data: choosing the signal components and selecting the order in exponential signal models," Proceedings of the IEEE, vol. 72, no. 2, pp. 230-233, 1984.

[19] J. F. Hauer, "The use of Prony analysis to determine modal content and equivalent models for measured power system response," in Proceedings of the IEEE/PES Symposium on Application of Eigenanalysis and Frequency Domain Methods for System Dynamic Performance, pp. 105-115, 1989.

[20] M.-C. Pai, "Closed-loop input shaping control of vibration in flexible structures via adaptive sliding mode control," Shock and Vibration, vol. 19, no. 2, pp. 221-233, 2012.

[21] J. Shan, H.-T. Liu, and D. Sun, "Slewing and vibration control of a single-link flexible manipulator by positive position feedback (PPF)," Mechatronics, vol. 15, no. 4, pp. 487-503, 2005.

[22] N. C. Singer and W. P. Seering, "Preshaping command inputs to reduce system vibration," Journal of Dynamic Systems, Measurement and Control, vol. 112, no. 1, pp. 76-82, 1990.

[23] R. Liu, Y. Gao, S. Yang, and Y. Yang, "Vibration control of the boom system of truck-mounted concrete pump based on constant-position commandless input shaping technique," Shock and Vibration, vol. 2015, Article ID 420935, 9 pages, 2015.

[24] W. Singhose, S. Derezinski, and N. Singer, "Extra-insensitive input shapers for controlling flexible spacecraft," Journal of Guidance, Control, and Dynamics, vol. 19, no. 2, pp. 385-391, 1996. 


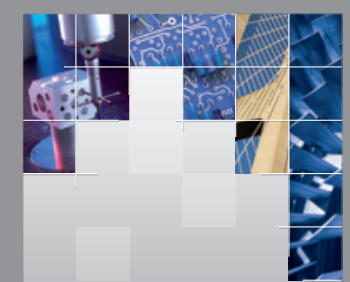

\section{Enfincering}
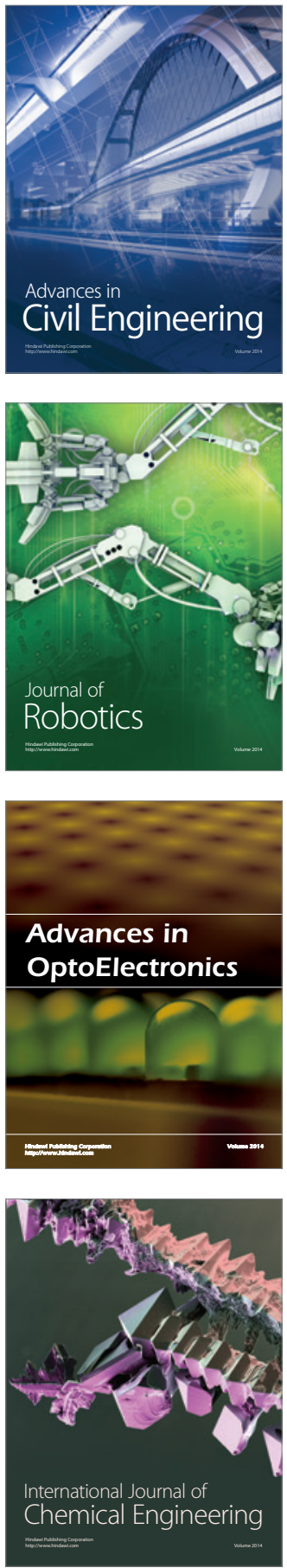

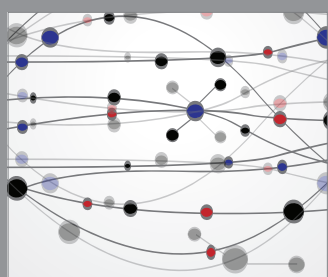

The Scientific World Journal

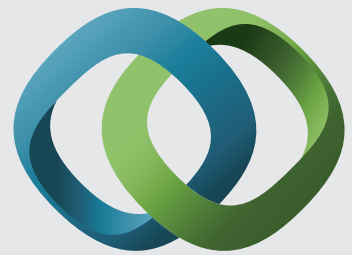

\section{Hindawi}

Submit your manuscripts at

http://www.hindawi.com
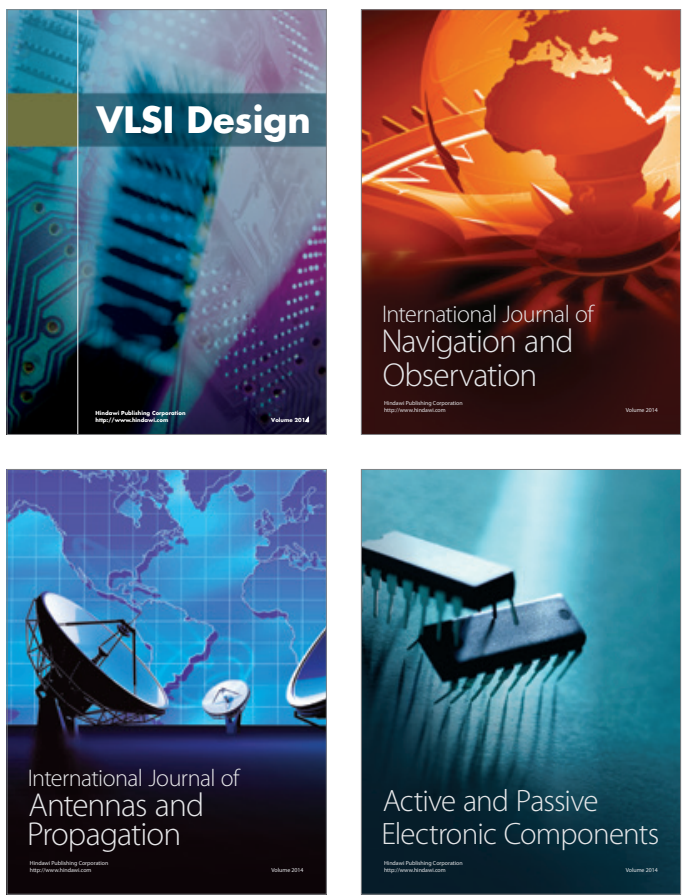
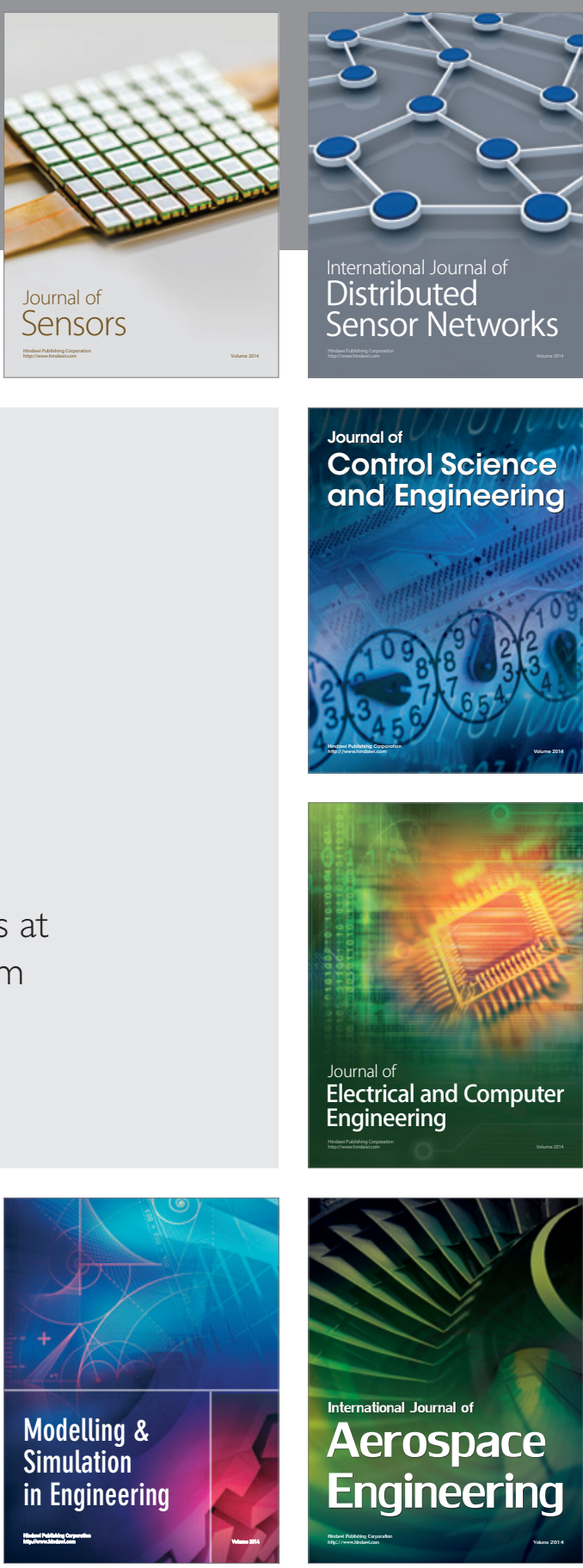

International Journal of

Distributed

Sensor Networks

Journal of

Control Science

and Engineering
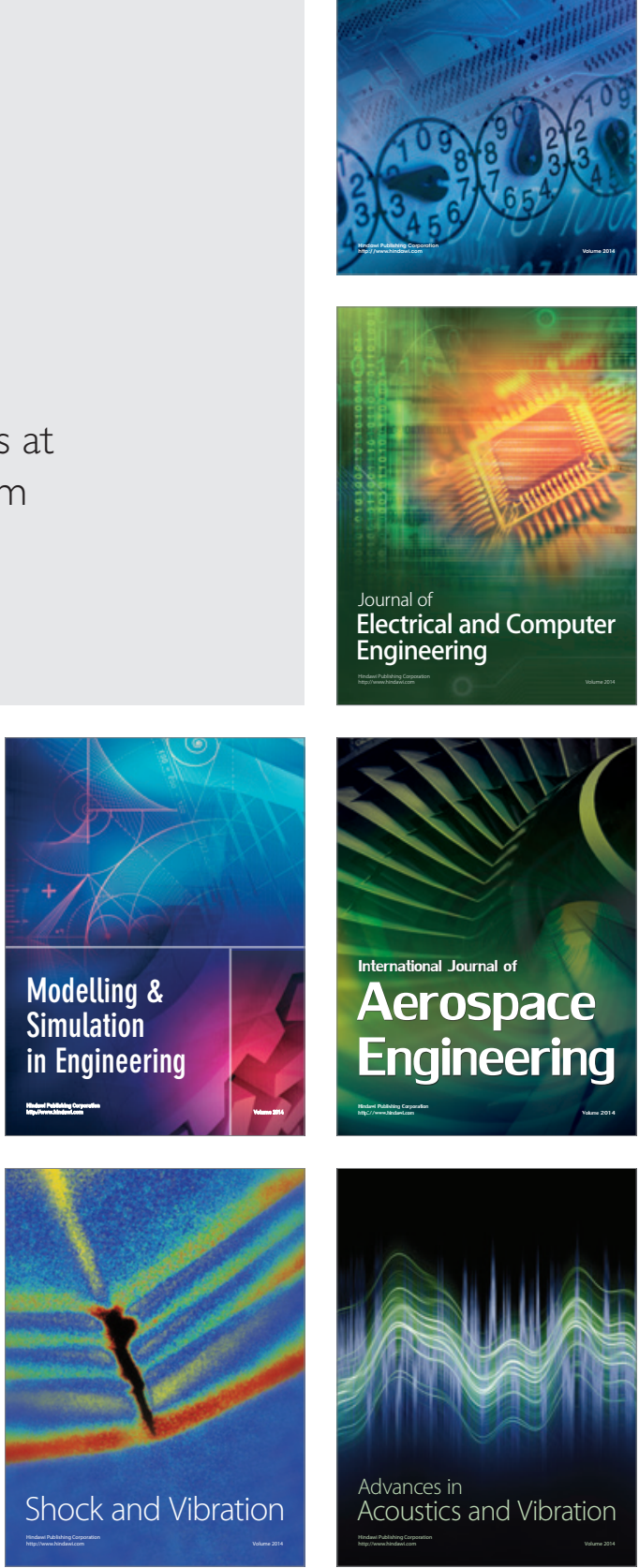